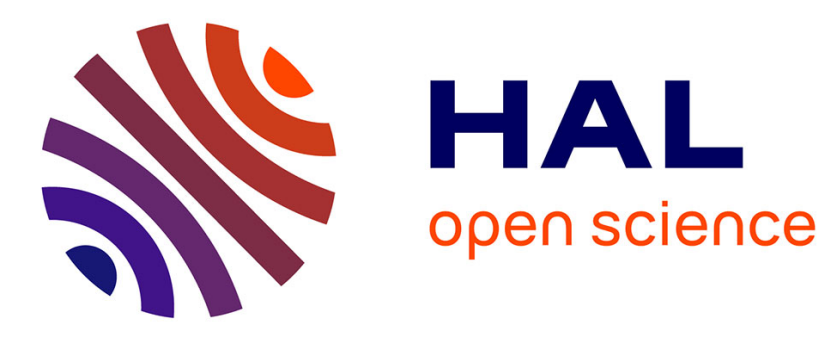

\title{
Raison statistique et catégories du droit de la santé
}

Isabelle Vacarie

\section{To cite this version:}

Isabelle Vacarie. Raison statistique et catégories du droit de la santé. Cahiers, Droit, Sciences \& Technologies, 2014, Statistiques et normes. Croiser les regards sur la formation du droit et la formation des connaissances, 4, pp.57-73. 10.4000/cdst.310 . hal-01655379

\section{HAL Id: hal-01655379 \\ https://hal.parisnanterre.fr/hal-01655379}

Submitted on 4 Jan 2018

HAL is a multi-disciplinary open access archive for the deposit and dissemination of scientific research documents, whether they are published or not. The documents may come from teaching and research institutions in France or abroad, or from public or private research centers.
L'archive ouverte pluridisciplinaire HAL, est destinée au dépôt et à la diffusion de documents scientifiques de niveau recherche, publiés ou non, émanant des établissements d'enseignement et de recherche français ou étrangers, des laboratoires publics ou privés. 


\title{
Raison statistique et catégories du droit de la santé
}

\author{
Isabel le Vacarie*
}

Résumé : Par touches successives, tant le législateur que le juge promeuvent une nouvelle pratique médicale davantage fondée sur les preuves scientifiques que sur l'expérience clinique du praticien, une " Evidence-based medicine ». En parallèle se développe une politique de couverture des frais de santé proportionnelle au service rendu par les diverses alternatives médicales pertinentes. Les outils de la statistique alimentent ces évolutions, qu'ils permettent une objectivation de la maladie à partir d'études menées sur un échantillon de personnes ou, par le calcul coûts-bénéfices, une quantification des utilités. Cette progression de la raison statistique au coeur même de la distribution des soins traduit un renouvellement des catégories de pensée. Prêter attention à ce renouvellement permet de comprendre l'organisation d'ensemble du droit de la santé, d'en saisir la dynamique. Elle est aussi la meilleure façon de souligner quelles sont, en retour, les limites qu'un système juridique tel que le nôtre fixe aux innovations politiques dans l'usage des statistiques.

Abstract: Law-makers as well as judges are sponsoring a new medical approach based on data analysis rather than clinical examination: an evidence-based medicine. In parallel, a policy of health coverage that is dependent on the efficienci of the respective medical options is being developed. Statistics supports these trends by promoting the quantitative description of illnesses and remedies and, in turn, allowing the cost-benefit analisis of medical treatments. The growing influence of statistics in public health is indicative of a renewed way of thinking. It is of paramount importance to pay attention to this shift of paradigm to understand the overall regulation of the health sector and its dynamics. It is also the best way to question the limitations our legal system may place on policy innovations based on the use of statistics.

* Professeur émérite à l'Université Paris Ouest Nanterre La Défense (isabelle.vacarie@u-paris10.fr). 
Les STATISTIQUES, écrit Alain Desrosières, constituent une forme d'objectivation du réel. Elles ne reflètent pas la réalité mais une manière de la voir. L'activité de quantification permet de constituer les faits sociaux en choses, de créer des objets macrosociaux sur lesquels prendre appui pour exprimer le monde et agir sur lui ${ }^{1}$.

Si cette façon de regarder le monde a trouvé un terrain privilégié au sein des sciences sociales, elle n'est pas propre à ce champ disciplinaire. La statistique est une discipline transversale, « une science passerelle » qui n'a plus d'objet privilégié mais qui propose « une méthode, utilisable par toutes les sciences de la nature et de l'homme, de production et de traitement de l'information à des fins de connaissance et de décision $»^{2}$. Ce mode de pensée a notamment trouvé place parmi les sciences de la santé. Ainsi est-il devenu l'une des composantes de la santé publique en suscitant la constitution d'une discipline, l'épidémiologie, qui, née au XIX $X^{e}$ siècle, n’a depuis cessé de se développer. Si les techniques du dénombrement furent d'abord utilisées pour analyser les épidémies, le raisonnement probabiliste donna ensuite aux épidémiologistes les moyens d'étendre leur champ d'étude aux autres facteurs de maladies. Pas à pas les statistiques les amenèrent à construire différents concepts tels que celui d'accident, de facteurs de risques ou de causes de mortalité sur lesquels prendre appui pour développer une politique de santé à l'échelle de la population ${ }^{3}$.

Dans un rapport récent, l'Académie des sciences souligne la progressive diversification des formes et des usages de la quantification ${ }^{4}$. Cette amplitude n'étonnera pas non plus car la statistique n'est pas une technologie univoque. Différentes approches historiques ont révélé la diversité des pratiques, selon les configurations dans lesquelles elles se déploient ${ }^{5}$. Dit autrement, les outils employés et les fonctions qui leur sont assignés participent directement de la façon de penser l'action publique ${ }^{6}$. Cette loi se vérifie tout particulièrement dans le domaine de la santé. Longtemps utilisés à des

1. A. Desrosières, La Politique des grands nombres. Histoire de la raison statistique (1999), La Découverte,

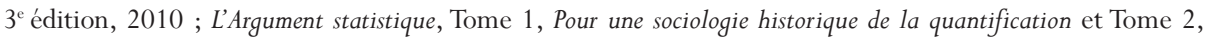
Gouverner par les nombres, Presses des Mines, 2008.

2. M. Armatte, La Science économique comme ingénierie. Quantification et modélisation, Paris, Presses des Mines, 2010, 345 p.

3. M. Foucault, Sécurité, territoire, population. Cours au Collège de France. 1977-1978, Seuil-Gallimard, 2004. Voir spécialement la leçon consacrée au gouvernement des populations, p. 57 et suivantes et celle qui analyse la naissance d'une nouvelle gouvernementalité, p. 341 et suivantes. Pour une histoire de l'épidémiologie et de ses usages sociaux et politiques, voir L. BERLIVET, « Déchiffrer la maladie », in Critique de la santé publique. Une approche anthropologique, sous la direction de J.-P. DOZON et D. FASsIN, Balland.

4. Académie des sciences, L'Épidémiologie humaine, conditions de son développement en France et rôle des mathématiques, sous la direction de A.-J. VALLERON, EDP sciences, 2006.

5. Sur l'histoire et la sociologie de la quantification, outre les ouvrages précités, voir Entretien avec A. DesrosièresetL. WolfF, Courrierdesstatistiques, $\mathrm{n}^{\circ} 127$, mai-août 2009.

6. A. Desrosıères, l'État, le marché et les statistiques, cinqfaçons d'agir surl'économie, Courrier des statistiques, n 95-96, décembre 2000 ; P. LASCOUMES, P. LE GALES, «L'action publique saisie par les instruments », in Gouverner par les instruments, Paris, Presses de Sciences Po, 2005, p. 11-44. 
fins explicatives, pour établir des corrélations statistiquement significatives entre deux phénomènes observables, les modèles mathématiques sont désormais mobilisés pour procéder à l'évaluation du service médical rendu tant par les actes médicaux répertoriés que par les produits de santé mis sur le marché. L'expression même de service rendu suggère un changement de l'angle sous lequel la santé est saisie : dans une perspective économique et non plus épidémiologique. Cet autre usage de la statistique est promu par une série de dispositions législatives qui ont conduit à la création d'une nouvelle institution, la Haute Autorité de santé (HAS), puis, par touches successives à l'élargissement de ses missions : de la détermination des bonnes pratiques médicales à l'évaluation de l'efficience des prestations, produits et technologies de santé pris en charge par l'assurance maladie?

A priori, ce type d'évaluation se présente sous un jour trop technique pour mériter que l'on s'y arrête. Plusieurs facteurs suggèrent néanmoins qu'il pourrait être, au contraire, le signe d'une révolution silencieuse de la construction juridique des relations médicales. Tout d'abord, la nature attribuée à cette institution : selon les propres termes de la loi, la HAS est une autorité à caractère scientifique. Ses évaluations bénéficient des qualités prêtées à toute démarche scientifique : une production objective, axiologiquement neutre, qui dit vrai, qui donne aux choses et aux situations dont elle parle un statut d'évidence, de contrainte logique. Ensuite, sa qualité d'autorité publique indépendante. Dès lors, ses avis et recommandations se voient reconnaître le poids que le système juridique accorde à ce type d'actes lorsqu'ils émanent d'une personne publique. Enfin l'objet sur lesquels porte les travaux de la HAS : la détermination des soins que la pathologie diagnostiquée rend utiles et de ceux qui méritent d'être pris en charge par un mécanisme d'assurance sociale. En d'autres termes, la manière dont le principe de protection de la santé est garanti.

Le moment semblait venu de s'intéresser aux usages de la statistique dans ce nouveau contexte, en faisant l'hypothèse que cette progression de la mesure au cœur même de la distribution des soins traduisait un changement des catégories de pensée prises pour référence : la maladie plutôt que l'individu malade ; l'efficience de l'acte ou du produit au lieu et place de sa seule qualité ; la rationalité de l'agent économique de préférence au couple droits-devoirs du sujet de droit. Autant de catégories auxquelles il y aurait lieu de prêter attention pour qui souhaite comprendre l'organisation d'ensemble du droit de la santés.

\footnotetext{
7. Code de la sécurité sociale, articles L. 161-37 et suivants et R. 161-70 et suivants.

8. Michel FoucAult invite à se livrer à ce type d'analyse dans les pages qu'il a consacré à l'histoire de la «gouvernementalité ». Il y résumait sa méthode en ces termes : « montrer qu'à partir de l'analyse relativement locale, relativement microscopique des formes de pouvoirs il était tout à fait possible [...] de rejoindre les problèmes généraux qui sont ceux de l'État, à condition justement que l'on [n'érige pas] l'État [en] une réalité transcendante dont l'histoire pourrait être faite à partir d'elle-même [...]. L'État comme manière de faire, l'État comme manière de penser », in Sécurité, territoire, population. Cours au Collège de France. 1977-1978, Seuil-Gallimard, 2004, p. 366.
} 
Pour qui veut également saisir la nature et les limites des innovations politiques dans l'usage des outils de la statistique'.

Dans cette perspective nous analyserons le processus qui a mené de l'explication à l'évaluation (I) pour ensuite examiner les recompositions qui en résultent dans cette région du droit (II).

\section{LE PROCESSUS}

Analyser le processus qui a mené de l'explication à l'évaluation revient à se demander « qu'est-ce qui fait qu'on se met à penser autrement ? $»^{10}$, quelles évolutions tant des outils de la statistique que de la manière de concevoir l'action publique ont initié ce mouvement?

À plus ample examen, un double mouvement se dessine. En premier lieu une objectivation de la maladie dans le but d'asseoir la pratique médicale sur une base scientifique (A) ; puis, en second lieu, une quantification des utilités, dans une perspective de santé publique et d'optimisation des dépenses d'assurance maladie (B).

\section{A. L'obJectivation de LA MALAdiE}

Dès sa création, le Haute Autorité de santé (HAS) eut pour tâche d'élaborer des recommandations de bonnes pratiques ${ }^{11}$. La détermination méthodique de la bonne pratique médicale s'inscrit dans un mouvement plus général venu des États-Unis qui recourt aux outils de la statistique pour saisir sous la variabilité des histoires personnelles, une histoire générique de la maladie, et pour faire de la pratique médicale

\footnotetext{
9. Suivant en cela la voie ouverte par Robert SALAIS dans ses travaux destinés à faire saisir les usages et mésusages de l'argument statistique. Voir « Usages et mésusages de l'argument statistique : le pilotage des politiques publiques par la performance », Revue française des affaires sociales, $\mathrm{n}^{\circ}$ 1-2, 2010, p. $129-147$; «La donnée n'est pas un donné, pour une analyse critique de l'évaluation chiffrée de la performance », Revue française d'administration publique, $\mathrm{n}^{\circ} 135,2010-3$, p. 497-515.

10. Voir également A. OGIEN, «La volonté de quantifier. Conceptions de la mesure de l'activité médicale », Annales. Histoire, sciences sociales, $\mathrm{n}^{\circ} 2,2000$, p. 283-312. Après avoir souligné la discontinuité des régimes de pensée, M. Foucault pose cette question : «Qu'est-ce qui fait que brusquement on se met à penser autrement ?». Une telle interrogation est essentielle pour comprendre sous quel régime nous vivons, dans quel type de rationalité, quel est notre ordre intellectuel, quelles sont nos structures mentales d'aujourd'hui (ouvrage précité). Sur les exigences de méthode qu'emporte cette question, M. FOUCAULT, L’Ordre du discours, Paris, Gallimard, 1971, p. 53 et suivantes.

11. A. Allouache et I. Vacarie, «Les bonnes pratiques professionnelles, le sens des mots », Journal d'économie médicale, numéro spécial consacré aux transformations des professions médicales, $\mathrm{n}^{\circ}$ 5, volume 29, 2011, p. 183-195.
} 
une pratique scientifique, fondée sur des preuves, une «Evidence-Based Medicine » ${ }^{12}$ Comment le droit participe-t-il à la constitution et à la consécration de ce processus ?

\section{De LA MÉTHOdE À LA BONNE MÉTHODE DE PREUVE}

Un historien des sciences, Harry Marks, a retracé l'histoire de la médecine des preuves, plus précisément de la méthode privilégiée pour établir ces preuves : l'expérimentation contrôlée ${ }^{13}$. Nous en retiendrons l'enseignement essentiel. Alors que la variabilité qui affecte tous les aspects du vivant semblait s'opposer à toute généralisation des résultats observés sur un nombre limité de cas, la statistique a transformé l'aléa en allié de la connaissance. Si le hasard a ses caprices, il a aussi ses lois : les lois de la probabilité. Prendre appui sur ces lois a permis de déterminer dans quelle mesure il est possible d'inférer des résultats observés dans le cadre d'une étude ou d'un essai une corrélation entre deux événements ou bien l'efficacité ou l'inutilité d'un traitement. Quel est le risque d'erreur, quel est l'intervalle de confiance ${ }^{14}$ ? En donnant une mesure de l'incertitude inhérente aux résultats expérimentaux, le calcul des probabilités fournissait la preuve de leur significativité et, par ricochet, de la confiance qui méritait de leur être accordée. D'abord utilisées pour passer au crible les affirmations des fabricants de produits de santé, quant aux effets bénéfiques de ceux qu'ils mettaient sur le marché, les expérimentations contrôlées furent ensuite étendues à la comparaison des stratégies médicales ${ }^{15}$.

Pour autant, ce type de calcul ne permet que d'établir des corrélations statistiquement significatives. Cette limite de l'inférence statistique a tout naturellement conduit à multiplier les études, à les comparer puis à élaborer diverses règles de bonne méthode destinées à apprécier si les résultats des études sont suffisamment convergents pour décider que les corrélations statistiques pouvaient être considérées comme apportant des éléments de preuve robustes. Après qu'ait été mise au point une méthode destinée à faire apparaître les liens statistiquement significatifs, ont été élaborées des règles de bonne méthode pour apprécier les mérites respectifs des différentes études, le niveau de preuve qu'elles apportent et décider quelles sont les lignes de conduite que l'on est autorisé à en tirer sur la plan pratique ${ }^{16}$.

12. Sur l'Evidence-Based Medicine, A. Fagot-Largeault, « Preuve et niveau de preuve dans les sciences biomédicales », in La Vérité dans les sciences, sous la direction de J.-P. ChangEUX, Paris, Odile Jacob, 2003, p. 215 et suivantes. F. FOURRIER, «Evidence-Based Medicine », in Dictionnaire de la pensée médicale, sous la direction de D. LECOURT, PUF, 2004.

13. H. MARKS, La Médecine des preuves. Histoire et Anthropologie des essais cliniques (1900-1990), Les Empêcheurs de penser en rond, 1999.

14. D. SChWARTZ, «Statistique et vérité », Journal de la société statistique de Paris, Tome 125, n 2, 1985, p. 74-83. A. FAGOT-LARGEAULT, «Épidémiologie et causalité », in L'Épidémiologie humaine, précité note (4).

15. Sur les usages de la statistique dans les expérimentations contrôlées, I. LÖWY, Essai clinique in Dictionnaire de la pensée médicale, précité note (12).

16. L. BeRLIVET, article précité note (3) ; A. Fagot-LARGEAULT, article précité note (12). 
La formulation par le législateur des différents principes auxquels toute expertise sanitaire doit répondre donne aujourd'hui un solide appui à cette médecine des preuves. Ces principes constituent en effet autant de règles de bonne méthode pour apprécier la qualité des études produites et le niveau de preuve. Ils garantissent en outre la validité de l'expertise du point de vue du droit. Quatre sont énumérés par le Code de la santé publique. Il s'agit des principes d'impartialité, de transparence, de pluralité et du contradictoire $^{17}$. Ces différents principes président désormais à l'élaboration des recommandations de bonnes pratiques émises par la Haute Autorité de santé.

\section{De La PRATIQUe À LA BONNE PRATIQUE DE LA MÉDECINE}

Dans l'autopsie qu'il fait de la contrainte associée aux normes juridiques, Paul Amselek distingue le commandement de la recommandation. Cette distinction souligne-t-il correspond à deux modes différents de l'art de diriger les conduites humaines. Ceux auxquels s'adressent les recommandations « possèdent par hypothèse même la faculté de s'en affranchir si cela leur paraît nécessaire ou plus opportun d'après leur propre appréciation du contexte ». Lorsque la personne exerce cette faculté de s'en affranchir elle n'a pas à motiver sa décision, par plus qu' elle n'est susceptible de sanction ${ }^{18}$.

Il s'observe que lorsque les recommandations portent sur les bonnes pratiques professionnelles, elles se prêtent à d'autres usages. Ce phénomène se vérifie tout particulièrement en présence des bonnes pratiques médicales. D’abord utilisées pour jauger la bonne exécution des obligations médicales, leur suivi devient aujourd'hui une composante de la politique de santé. De référence pour l'action, la médecine des preuves se mue en objectif à atteindre. Suivre ce mouvement permettra de suivre le cours d'un glissement conceptuel.

Une association reprochait à la Haute Autorité de santé son manque d'impartialité dans l'élaboration d'une recommandation relative au traitement médicamenteux du diabète, en raison de la présence au sein du groupe de travail chargé de sa rédaction, d'experts médicaux qui entretenaient des liens d'intérêts avec les entreprises pharmaceutiques intéressées. Le Conseil d'État fut saisi après que la Haute Autorité de santé ait manifesté son refus de retirer cette recommandation. Dans cette affaire jugée le 12 avril 2011, les conclusions du rapporteur public rendent parfaitement compte de l'interprétation téléologique qui a été la sienne pour qualifier ces recommandations. Bien que dépourvue de toute valeur contraignante propre, les recommandations émises par la Haute Autorité de santé n’en ont

\footnotetext{
17. Article L. 1452-1 modifié par la loi du 29 décembre 2011 relative à la sécurité sanitaire. Sur les apports de l'analyse juridique à l'expertise, voir la conclusion prospective de R. ENCINAS DE MunAGORRI et de O. LECLERC, in Expertise et gouvernance du changement climatique, Paris, LGDJ, « Droit et société », 2009. 18. P. AMSELEK, «Analyse de la contrainte associée aux normes juridiques », in La Force normative, C. THIBIERGE et alii, Paris, LGDJ, 2009, p. 3-11.
} 
pas moins vocation à servir de référence pour juger si le médecin a correctement exécuté les devoirs que lui dicte la déontologie, plus particulièrement celui d'assurer au patient des soins fondés sur les données acquises de la science. Leur normativité tient à ce qu'elles ont «valeur de préjugé » pour l'autorité disciplinaire ${ }^{19}$. Dès lors parait-il opportun que « le juge administratif puisse en amont s'assurer que ces recommandations ne font pas la part belle à tel ou tel intérêt particulier ». Cette liaison opérée entre recommandation de bonnes pratiques et déontologie médicale a conduit le Conseil d'État à conclure que les premières « doivent être regardée comme des décisions administratives faisant grief susceptibles de faire l'objet d'un recours pour excès de pouvoir $»^{20}$.

En raison de cette fonction d'instrument de mesure, de référence pour l'action, le rapporteur public n'excluait pas qu'à l'occasion de poursuites disciplinaires, le médecin puisse contester leur validité, et plus précisément leur conformité aux données acquises de la science. Cette interprétation peut ne pas convaincre car elle suppose que les données de la science préexisteraient à leur formulation par la Haute Autorité de santé. Est-ce que le processus n'est pas exactement inverse ? Pour reprendre les mots de Robert Salais, « les données ne sont pas un donné, elles sont construites et sélectionnées » et l'élaboration des recommandations correspond très précisément à cette opération de sélection ${ }^{21}$. Dans sa décision, le Conseil d'État emploie d'ailleurs une formule éclairante : « les données de la science telles qu'elles ressortent notamment des recommandations ». Cela ne signifie pas que le médecin n'aura aucun moyen de les discuter. Mais sa contestation ne portera pas sur leur non-conformité à des données qui ne leur préexistent pas ; il leur reprochera plutôt de n'avoir pas été élaborées dans le respect des règles de bonne méthode pour apprécier la qualité des études produites et le niveau de preuve, autant de règles constitutives des principes auxquels doit répondre toute expertise sanitaire.

Un basculement s'opère lorsque les pratiques médicales fondées sur des preuves deviennent la matrice d'une politique publique incitative.

19. La normativité d'un énoncé, souligne A. JEAMMAUD, « réside dans la vocation et l'aptitude de cet énoncé à servir de référence pour dire et déterminer, en présence de situations concrètes, comment les choses doivent être, dans une opération d'évaluation des données de la situation considérée. Évaluation ex ante, en vue d'agir [...] ou ex post, en vue de réagir ou de juger », in «La règle de droit comme modèle », Recueil Dalloz, 1990, chronique 199 ; «Le concept d'effectivité du droit » in L'effectivité du droit du travail : à quelle condition?, Université de Montesquieu-Bordeaux IV, Comptrasec, Actes du séminaire international de 2005, p. 33-54. É. MiLlaRD, «Qu'est-ce qu' une norme juridique ?», Cahiers du Conseil constitutionnel, n 21, janvier 2007 (dossier consacré à la normativité).

20. Conseil d'État, 27 avril 2011, Association Formindep, $\mathrm{n}^{\circ}$ 334396, conclusions C. LANDAIS, L'Actualité juridique de droit administratif (AJDA), 2011, p. 1326 ; note J. PEIGNÉ, Revue de droit sanitaire et social (RDSS), $\mathrm{n}^{\circ}$ 3, 2011, p. 483.P. LOISEAU, « La force normative des recommandations de bonnes pratiques médicales », in La force normative, précité note (18), p. 613-622. F. SAVONITTO, « Les recommandations de bonne pratique de la Haute Autorité de Santé », Revue français de droit administratif (RFDA), 2012, p. 471.

21. R. SALAIS, « La donnée n'est pas un donné », article précité note (9). 
Divers dispositifs dont la source se trouve dans le Code de la sécurité sociale nourrissent ce mouvement ${ }^{22}$. Nous n'en prendrons qu'une seule illustration puisée dans la dernière convention nationale des médecins ${ }^{23}$. Trois éléments composent le système institué par les partenaires conventionnels. En premier lieu, les parties signataires arrêtent, d'un commun accord, une liste d'indicateurs qui répond «à des objectifs de santé publique et d'optimisation des dépenses ». Ces indicateurs, est-il précisé, sont fixés en cohérence avec la loi de santé publique et s'appuient tant sur les protocoles et référentiels émis par la Haute Autorité de santé que sur les recommandations internationales « afin d'être adaptés aux données acquises de la science ». L'accord porte ensuite sur la manière de valoriser financièrement l'implication des médecins dans la réalisation de ces objectifs : au paiement de l'acte, s'ajoute une rétribution proportionnelle aux objectifs atteints par chaque médecin. Le texte de la convention précise enfin que ces dispositions s'appliquent à l'ensemble des médecins conventionnés, à l'exception de ceux qui auraient manifesté leur refus dans les trois mois de la publication de la convention. Sans qu'il soit nécessaire d'entrer davantage dans le détail du mécanisme, retenons son trait caractéristique : donner à chaque médecin un intérêt financier à suivre la pratique préconisée par les indicateurs de performance, à conformer ses prescriptions à celles qui sont énoncées comme répondant à une prise en charge optimale de la maladie ${ }^{24}$.

La diffusion de la démarche statistique dans le domaine de la médecine emporte ainsi une succession de glissements conceptuels. Le malade pris individuellement et engagé dans un « colloque singulier » avec son médecin tend à s' effacer devant la maladie telle qu'elle est saisie et mise en forme par les études scientifiques internationales. Le crédit accordé à l'expérimentation prend progressivement le pas sur l'expérience quotidienne de la pratique clinique. Le savoir médical se trouve externalisé. L'objectivation de la maladie et le développement d'une méthode de preuve emportent à leur tour une standardisation, une normalisation de la pratique médicale.

Autant de glissements qui n'en sont pas moins motivés par les vertus prêtées aux outils de la statistique : donner la mesure de l'incertitude, et permettre ainsi de placer la pratique médicale sur des bases scientifiques.

Un pas d'une toute autre nature est franchi lorsque les mathématiques sont utilisées pour calculer le rapport coût-bénéfice et quantifier les utilités.

22. Voir les articles L. 162-12-17 et suivants du Code de la sécurité sociale consacrés aux accords de bon usage et contrats de bonne pratique des soins

23. Voir l'arrêté du 22 septembre 2011 portant approbation de la convention nationale des médecins conclue le 26 juillet 2011, sous-titre 5, intitulé : «Compléter la rémunération pour valoriser la qualité des pratiques médicales et l'efficience » (articles 25 et suivants de la convention).

24. A. Allouache, A.-S. Ginon et alii, «Une médecine « responsable» in Droit, sciences et techniques quelles responsabilités? Paris, Lexis Nexis, 2011, p. 509-522. B. DoRMONT, «Le paiement à la performance : contraire à l'éthique ou au service de la santé publique ? », Les Tribunes de la santé, 2013/3, n 40, p. 53-61. 


\section{B. LA QUANTIFICATION DES UTILITÉS}

Outre l'élaboration des recommandations de bonne pratique, la loi charge la Haute Autorité de santé de «procéder à l'évaluation périodique du service attendu des produits, actes ou prestations de santé et du service qu'ils rendent ». Cette évaluation du service rendu joue un rôle-clé pour l'ensemble des acteurs du système dans la mesure où elle prédétermine le statut de chaque produit, acte ou prestation. L'avis rendu par la Haute Autorité de santé précise en effet les textes, contribue à l'élaboration de toutes les décisions qui fixent ce statut : prix ou tarif, inscription sur la liste des produits ou des actes remboursables et taux de remboursement.

Depuis la publication de la loi de financement de la sécurité sociale pour 2012 (loi du 21 décembre 2011), le législateur invite expressément la Haute autorité de santé à évaluer leur efficience. Cette évaluation va jusqu'à être requise lorsque « le produit ou la technologie a ou est susceptible d'avoir un impact significatif sur les dépenses de l'assurance maladie ». Un décret en date du 2 octobre 2012 précise le type d'analyse à laquelle se livre la Commission d'évaluation économique : « une analyse du rapport entre les coûts engagés et les bénéfices attendus ou observés pour la santé et la qualité de vie des personnes concernées $»^{25}$.

La promotion de ce type d'analyse coûts-bénéfices retient l'attention à un double titre. D'une part, elle introduit explicitement la référence aux coûts du traitement dans les décisions de remboursement de l'assurance maladie qui correspondent à des dépenses socialisées. D’autre part, elle met en rapport des éléments a priori incommensurables ; si les coûts engagés sont susceptibles d'une expression monétaire, les bénéfices attendus pour la santé s' expriment en unités naturelles (nombre de semaines, de mois ou d'années de vie gagnée) ; la qualité de vie dépend quant à elle des préférences propres à chaque personne concernée. La force, et peut-être la magie, de la statistique consiste à fournir des outils permettant de mettre en chiffres, ce qui ne semblait pouvoir s'exprimer que par des mots. Les ouvrages de statistiques rendent compte des divers modèles qui ont été progressivement construits dans le but de calculer, à partir d'un échantillon limité de personnes, une médiane de survie, ou encore de quantifier la qualité de vie que procure tel ou tel traitement ${ }^{26}$. Un autre monde est ainsi créé : un monde d'indicateurs chiffrés, tous commensurables, qui se prêtent à une évaluation en termes de service rendu ${ }^{27}$.

25. Loi n ${ }^{\circ}$ 2011-1906 du 21 décembre 2011 de financement de la sécurité sociale pour 2012, article 47 qui modifie l'article L. 161-37 du Code de la sécurité sociale. Décret n 2012-1116 du 2 octobre 2012 relatif aux missions médico-économiques de la Haute Autorité de santé qui complète l'article R. 161-71 et suivants du Code de la sécurité sociale. Sur l'introduction du calcul économique dans le domaine de la santé, C. LE PEN, «Calcul économique et décision en santé », in Traité d'économie et de gestion de la santé, sous la direction de P.-L. Bras, G. de Pouvourville et D. Tabuteau, Presses de la Fondation nationale des sciences politiques, 2009, p. 63-73.

26. Voir l'article de C. Le PEN précité.

27. Voir sur le site de la Haute Autorité de santé, Le Guide méthodologique pour l'évaluation économique à la Haute Autorité de santé, octobre 2011. 
Cette évaluation, faut-il le préciser, implique la comparaison. Dans les situations où l'avis de la Haute Autorité de santé est désormais requis, il est précisé que cet avis se fonde sur une analyse comparative entre les différentes alternatives médicalement pertinentes. Un basculement se produirait si la comparaison allait au-delà pour porter sur différentes pathologies. Par exemple, est-ce qu'un euro « investi » dans la réduction de la glycémie chez les patients diabétiques est plus « coût-efficace » qu'un euro investi dans la réduction de la tension artérielle chez les patients hypertendus sévères ? Tel ne semble pas avoir été le projet du Parlement lors du vote de la loi de financement de la sécurité sociale pour 2012. Pour autant le texte du décret d'octobre 2012 énonce, dans des termes très généraux, que la HAS contribue à la comparaison et à la hiérarchisation des stratégies de prévention et de soins, dans un objectif de santé publique et d'optimisation des dépenses de santé ${ }^{28}$. Ainsi que cela a été très justement noté, « une efficience peut en cacher une autre $»^{29}$.

S'il fallait rechercher la théorie dont se sont inspirés les auteurs de ces diverses dispositions, sans doute faudrait-il se tourner vers la théorie du calcul économique public ${ }^{30}$. On retrouve en effet dans les textes la méthode prônée par cette théorie : quantifier aussi exhaustivement que possible les besoins satisfaits par le service proposé. La légitimité intellectuelle de cette méthode tient à la fonction que cette théorie attribue aujourd'hui à l'État : répondre à des besoins complexes, dans un monde où le nombre de biens et la concurrence entre eux ne font que croître. Dans ce contexte, la quantification des utilités est présentée comme le type de calcul qui répond le mieux à un certain optimum social, qui permet que le choix public soit d'un point de vue social, le plus utile. Autrement formulé, ce type de calcul serait la plus sûre façon de réaliser le bien commun, il deviendrait une composante de l'intérêt général. C'est, à n'en pas douter, ce qui donne aujourd'hui à cette théorie sa puissance argumentative. C'est également ce qui permet de comprendre sa diffusion dans le droit de la sécurité sociale.

Quelle que soit la pertinence de cette théorie, observons que les modèles de calcul qui président à l'évaluation du service rendu n'en restent pas moins des artéfacts. Bernard Wallizer souligne leur effet de réalité, leur performativité. « Dans sa fonction rhétorique, la principale qualité d'un modèle est sa faculté à être accepté comme une connaissance valide par les agents concernés $»^{31}$. Ici encore, bien que la Haute Autorité de santé ne formule qu'un avis, son activité de quantification sert de point d'appui pour établir la norme.

\footnotetext{
28. Article R. 161-71, $4^{\circ}$ ajouté par le décret du 2 octobre 2012.

29. C. LE PEN, « Une efficience peut en cacher une autre », La Lettre du Collège des économistes de la santé, $\mathrm{n}^{\circ} 2$, juin 2012.

30. Sur cette théorie, R. GUESNERIE, «De l'utilité du Calcul économique public, Économie et prévision », $\mathrm{n}^{\circ} 175-176,2006 / 4-5$.

31. B. WALlizer, «Les fonctions des modèles économiques », in Leçons de philosophie économique, Tome III, Sciences économiques et philosophie des sciences, Paris, Economica, 2007, p. 285-302.
} 
Elle fournit des chiffres de référence pour déterminer le panier des soins remboursables et par voie de conséquence les termes dans lesquels l'accès aux soins est assuré.

\section{LES RECOMPOSITIONS}

Recommandations de bonnes pratiques, évaluation du service rendu, dispositifs incitatifs, autant d'outils juridiques qui ne peuvent rester sans incidence sur la construction d'ensemble du droit de la santé.

Tandis que leur objet - les actes et produits de santé - suscite un emboîtement du droit de la santé et du droit de l'assurance maladie (A), leurs ressorts - les indicateurs chiffrés alimentent l'image d'un droit dont la fonction serait « une fonction promotionnelle » (B).

\section{A. L'EMBOîTEMENT DU DROIT DE LA SANTÉ ET DU DROIT DE L'ASSURANCE MALADIE}

Les différentes dispositions qui ont progressivement généralisées l'analyse économique tant des produits que des stratégies de santé figurent dans le Code de la sécurité sociale. À cela rien de surprenant car toutes ont été adoptées pour répondre à l'objectif d'équilibre financier de la sécurité sociale. Dans un même mouvement, cet objectif, auquel le Conseil constitutionnel attribue désormais valeur constitutionnelle, a suscité et justifié la mise en ouvre de divers procédés de régulation des dépenses. L'idée s'est en effet imposé qu'à l'image des organismes vivants, le système de sécurité sociale devait être conçu de telle manière qu'il développe ses propres techniques d'équilibre ${ }^{32}$. Sur ce chemin, l'évaluation du service rendu est devenu l'un des points forts de la régulation des dépenses de la branche assurance maladie ${ }^{33}$. Dans une telle configuration, la norme se fixe et joue son rôle opératoire à partir du calcul. « C' est le normal qui est premier et c'est la norme qui s'en déduit $»^{34}$. Celle-ci est découverte dans les choses elles-mêmes,

32. Sur les formes nouvelles prises par la fonction de régulation dans les sociétés contemporaines,

J. Chevallier, «La Régulation juridique en question », Droit et société, n 49, 2001-3, p. 827-846. ; J.-M. LemoYne de Forges, «L'État et la santé, l'épreuve de la post-modernité », in Mélanges en l'honneur du professeur Jacques Chevallier, Paris, LGDJ, 2013, p. 581-593.

33. R. SALAIS souligne en ces termes l'ambivalence de l'évaluation dans un tel contexte : « L'évaluation de la performance quantifiée se trouver internalisée, à la fois comme référence et comme objectif, dans le processus de l'action. Une sorte de "court-circuit" se produit entre l'évaluation comme référence et la performance comme objectif » (article précité note 9).

34. Pour M. Foucault ce mode de fixation de la norme correspond à une nouvelle économie du pouvoir, à une opération de normalisation au sens strict du terme (ouvrage précité, note 3). Voir également les analyses de D. LOCHAK sur « La dissolution du normatif dans la normalité, Droit, normalité et normalisation », Curapp, Le Droit en procès, Paris, PUF, 1983, p. 54. 
elle appartient au seul monde des faits, elle est unidimensionnelle ${ }^{35}$. S'opère « une suture sans faille entre jugement des faits et jugement de valeurs $»^{36}$.

Dans la mesure où l'assurance maladie est une assurance sociale destinée à couvrir les charges de maladie, on pouvait penser que la régulation de ses dépenses était susceptible d'avoir des répercussions sur le taux de participation des assurés, sans rejaillir pour autant sur la construction juridique des soins. Tel n'est pourtant pas le cas en raison des choses sur lesquelles porte l'activité de régulation : la fixation du prix des médicaments et du tarif des dispositifs médicaux ; la détermination du panier des soins remboursables et du meilleur parcours de soins en fonction de la pathologie diagnostiquée ; ou encore celles des mesures de prévention qui méritent d'être privilégiées. Toutes choses qui structurent de bout en bout les relations de santé.

Nous verrons plus loin de quelle façon cette démarche est susceptible d'entrer en tension avec la liberté de prescription du médecin. Plus fondamentalement, elle part d'une conception de la santé à l'opposé de celle qui a nourri le développement de la branche du droit homonyme, plus particulièrement depuis la loi du 4 mars 2002 relative aux droits des patients.

Pour la personne individuellement considérée, soulignait Georges Canguilhem, santé et maladie n'ont aucune existence par elles-mêmes : ce sont des catégories de jugement. L'individu se déclare malade par un jugement comparatif porté sur son histoire propre, par contraste avec un état antérieur qu'il jugeait sain. S'il qualifie de pathologique un état de son organisme n'est-ce pas qu'il prend conscience de quelque rétrécissement de ses anciennes possibilités ? Il attend du médecin qu'il les rétablisse. Dès lors, la thérapeutique ne saurait se présenter comme simple application de normes statistiquement élaborées. Ainsi s'explique que, dans la tradition déontologique, le médecin doive à son patient les soins les plus appropriés en la circonstance. La maladie et son traitement constituent un événement singulier. Tout l'art du médecin est alors de savoir utiliser la variété des moyens disponibles pour répondre à ce cas unique ${ }^{37}$. À son tour la loi du 4 mars 2002, après avoir classé le droit à la protection de la santé parmi les droits fondamentaux dont toute personne est titulaire, a pris soin d'ajouter, en chapeau du Code de la santé publique, que ce droit «doit être mis en ouvre par tous moyens disponibles ». Toute personne prend «les décisions concernant sa santé », précise-t-elle ensuite ${ }^{38}$.

\footnotetext{
35. Sur les mirages de la quantification et les pièges de l'autoréférence, A. SuPIOT, L'Esprit de Philadelphie. La justice sociale face au marché total, Édition du Seuil, Paris, 2010, p. 75 et suivantes.

36. R. CAStel, «Savoir d'expertise et production de normes», in F. Chazel et J. Commaille (sous la direction de), Normes juridiques et régulation sociale, Paris, LGDJ, « Droit et société », 1991, p. 177-188.

37. Sur la notion de clinique, voir « Médecine et anthropologie», sous la direction de G. BarRoux, Corpus, revue de philosophie, $\mathrm{n}^{\circ}$ 54, 2008, 180 p. Également, La mort de la clinique ?, Paris, PUF, « Quadrige », 2009, 150 p.

38. Articles L. 1110-1 et L. 1111-4 du Code de la santé publique
} 
La méthodologie adoptée pour établir les bonnes pratiques, l’idée de panier de soins, reposent sur une toute autre représentation de la santé et de la maladie. La maladie est détachée de l'individu malade. Le normal et le pathologique deviennent des catégories statistiques. En prenant pour illustration les constantes biologiques, il a pu être montré de quelle manière le chiffre à partir duquel ces constantes sont anormales correspond à une décision. « La conception actuelle de la médecine est organisée autour de la norme biologique identifiée à un fait objectif supposé définir la santé $»^{39}$. Les objectifs portent sur des pathologies et des déterminants affectant des populations et des sous-populations construites par le raisonnement mathématique.

Seule cette représentation de la santé et de la maladie, du normal et du pathologique dissociée de la personne malade permet de penser que les soins dont la prise en charge relève de la solidarité nationale sont déterminables rationnellement, indépendamment de la singularité de chacun.

Se profile alors une autre approche des conditions dont dépend la garantie du droit à la protection de la santé : non plus en termes de prérogatives juridiques dont chacun serait titulaire, mais de parcours de soins et de niveau de prise en charge arrêtés pour chaque pathologie, à partir d'une évaluation régulière du service rendu par les différents actes et produits sur le marché. Autrement formulé, l'objectivation de la maladie a pour corollaire une objectivation des formes de réalisation du droit fondamental à la protection de la santét ${ }^{40}$.

De fins connaisseurs du système de santé prônent le rapprochement du droit de la santé et du droit de la sécurité sociale ${ }^{41}$. À n'en pas douter le mouvement est en cours : l'assurance maladie devient une composante du droit de la santé, mais, faut-il aussitôt ajouter, d'un droit de la santé reposant sur des catégories de pensées différentes de celles à partir desquelles il s'est historiquement construit. La confrontation des premières et des secondes illustre cette recomposition tout en soulignant les limites qu' elle rencontre. En témoigne le contentieux suscité par les dispositifs incitatifs dans leur vocation à promouvoir les bonnes pratiques.

39. Dictionnaire de la pensée médicale, sous la direction de D. LECOURT, Paris, PUF, 2004.

40. T. GRÜNDLER, «Effectivité, efficacité, efficience, l'exemple du « droit de la santé », in À la recherche de l'effectivité des droits de l'homme, sous la direction de V. CHAMPEIL-DesPLATS et D. LOCHAK, Nanterre, Presses universitaires de Paris 10, 2008, p. 31-40 ; Sur l'articulation entre totalisations statistiques et souci du particulier, voir N. DodieR, «Les sciences sociales face à la raison statistique (notre critique) », Annales. Histoire, sciences sociales, $\mathrm{n}^{\circ} 2$, 1996, p. 409-428, spéc. p. 418 et s.

41. D. TABUTEAU, « Code de la santé publique ou Code de la santé ? », Revue de droit sanitaire et social (RDSS), 2008, p. 416 ; «Droit de la santé et droit de l'assurance maladie », Revue de droit sanitaire et social (RDSS), 2008, p. 878 ; Démocratie sanitaire, Les nouveaux défis de la politique de santé, Paris, Odile Jacob, 2013, 290 p. 


\section{B. LA FONCTION PROMOTIONNELLE ASSIGNÉE AU DROIT}

«L'activité promotionnelle de l'État», Norberto Bobbio emploie cette expression pour nommer la politique législative qui consiste à encourager ou décourager certaines pratiques en donnant au destinataire de la règle un intérêt financier (gain ou coût) à adopter la conduite recherchée par les auteurs de la règle ${ }^{42}$. Deux décisions emblématiques montrent comment et pourquoi le développement de cette politique est susceptible d'entrer en tension avec les préceptes de la déontologie.

\section{Deux corpus de textes}

Nous avons précédemment évoqué l'instauration d'une nouvelle forme de rémunération des médecins libéraux qualifiée de « rémunération à la performance ». Directement versée aux médecins par les caisses de sécurité sociale, elle a pour vocation de rétribuer l'implication des médecins libéraux dans la réalisation d'un ensemble d'objectifs de santé publique et d'optimisation des prescriptions. Ces objectifs sont chiffrés à l'échelle de la population, puis ils sont déclinés, pour chaque médecin, à la taille de sa patientèle. Aussi trouve-t-on dans la convention nationale des médecins une batterie d'indicateurs qui sont simultanément destinés à formuler les objectifs et mesurer leur réalisation. La somme qui sera effectivement versée au médecin sera proportionnelle à ceux qu'il aura personnellement atteints ${ }^{43}$.

Ce mode de réalisation des objectifs publics par le truchement des actions individuelles n'est pas propre à la France. Il se développe dans un grand nombre de pays européens, essentiellement à partir du modèle anglais ${ }^{44}$. Dès lors ne s'étonnera-t-on pas que ce soit la High Court of Justice qui, la première, ait interrogé la Cour de Justice de l'Union européenne sur la compatibilité d'un système d'incitations financières mis en ouvre par les autorités nationales avec les dispositions du droit de l'Union destinées à protéger les principes déontologiques fondamentaux, au premier rang desquels figurent la liberté de prescription. Le renvoi préjudiciel portait sur la lecture que les juges nationaux devaient faire de la directive instituant un code communautaire des médicaments ${ }^{45}$. Était plus précisément en cause la signification de l'article 94 qui interdit de promettre une prime ou un avantage pécuniaire aux personnes habilitées à prescrire ou à délivrer ces produits. Aux yeux de la Cour, il résulte de l'économie générale de la directive que cette disposition vise la promotion commerciale des médicaments. Dès lors concerne-t-elle au

42. N. BobBIO, Essai de théorie du droit, Paris, LGDJ, 1998, p. 65 ; De la structure à la fonction. Nouveaux essais de théorie du droit, traduit et présenté par D. SoldINI, Paris, Éditions Dalloz, 2012, 186 p.

43. Voir particulièrement les articles 26 et suivants de la convention médicale.

44. P.-L. Bras, «Le contrat d'amélioration des pratiques individuelles à la lumière de l'expérience anglaise », Journal d'économie médicale, numéro spécial précité note (11), p. 216-230.

45. Directive $\mathrm{n}^{\circ}$ 2001-83 instituant un code communautaire relatif aux médicaments à usage humain. 
premier chef l'industrie pharmaceutique lorsqu'elle déploie des activités de promotion des médicaments tant auprès des médecins que des pharmaciens. Par contre, elle n’est pas applicable aux autorités publiques dans la mesure où ces dernières ne poursuivent ni but lucratif, ni but commercial lorsqu'elles développent une politique de santé. « Un système d'incitations financières relevant d'une telle politique ne saurait être considéré comme s'inscrivant dans le cadre de la promotion commerciale des médicaments ». Il fait au contraire partie des mesures qu'il est loisible à un État d'adopter dans l'exercice de ses responsabilités en matière de santé. Pour autant, poursuit la Cour, un tel dispositif ne saurait délier le médecin de ce que lui dicte la déontologie : « un médecin prescripteur est tenu d'un point de vue déontologique, de ne pas prescrire un médicament donné si celui-ci ne convient pas au traitement thérapeutique de son patient, et ce nonobstant l'existence d'incitations financières publiques à la prescription de ce médicament $»^{46}$.

À son tour, le Conseil d'État a affirmé dans des termes très généraux que le contrat qui s'établissait entre le médecin et les caisses de sécurité sociale «ne saurait avoir pour effet de dispenser le médecin du respect des règles qui s'imposent à lui en vertu du Code de déontologie médicale et notamment de celles qui, comme la liberté de prescription, ont été instituées dans l'intérêt des patients $\gg^{47}$. Si les réponses tant de la Cour de Justice que du Conseil d'État ne surprennent pas, le seul fait que l'une et l'autre de ces juridictions aient réaffirmé le respect par tout médecin des principes déontologiques en présence d'indicateurs de performance souligne que ces deux types de normes reposent sur des conceptions différentes des rapports entre droit et action.

\section{DEUX CONCEPTIONS DES RAPPORTS ENTRE DROIT ET ACTION}

Lorsque sont évoqués les rapports entre la règle et sa réalisation concrète, il s'agit toujours de souligner l'absence d'automatisme entre l'une et l'autre ${ }^{48}$. Là repose le paradoxe de la règle pratique observe Philippe Coppens : « elle nous oblige à agir d'une manière plutôt que d'une autre tandis qu' elle risque pourtant à tout instant de ne pas être suivie ». Par quelle voie « favoriser son efficacité dans l'orientation du choix d'action du sujet de droit $»^{49}$ ? Les travaux de Norberto Bobbio ont montré la diversité des procédés

46. Cour de Justice de l'Union européenne, 22 avril 2010, affaire C-62 :09, Association of the British Pharma Medical Industry contre Medecinas and Healthcare Produits Regulatory Agency ; M. AubERT, E. BroussY, F. DONNAT, chronique de la jurisprudence de la Cour de Justice de l'Union européenne, L'Actualité juridique de droit administratif(AJDA), 2010, p. 1578 ; C. MASCRET, Revue de droit sanitaire et social (RDSS), 2010, p. 765.

47. Conseil d'État, 7 avril 2011, n 329069, 329174, 332835.

48. A. Jeammaud, «Les règles juridiques et l'action », Recueil Dalloz, 1993, chronique, p. 207. Voir également V. Champeil-Desplats, «Effectivité et droits de l'homme, approche théorique », qui s'interroge sur la façon de «penser l'écart », in À la recherche de l'effectivité des droits de l'homme, sous la direction de V. Champeil-Desplats et D. Lochak, Nanterre, Presses universitaires de Paris 10, 2008, p. 11-25.

49. $\mathrm{Ph}$. Coppens, «La sanction de la règle juridique : de la contrainte à l'incitation », in La sanction : la lecture des économistes et des juristes, sous la direction d'Y. CHAPUT, Bruxelles, Bruylant, 2011, p. 27-47. 
possibles : parfois seule la transgression suscite une réaction juridique par le canal du prononcé d'une peine ou de toute autre sanction juridique ; parfois au contraire le dispositif juridique encourage financièrement la conformation des pratiques à la règle ${ }^{50}$.

Ce sont très précisément ces deux façons différentes d'encadrer l'activité médicale qui distinguent déontologie et indicateurs de performance. Dans le modèle déontologique, le respect des devoirs qui s'imposent à tout médecin n'emporte pas de réaction de l'ordre juridique. Au contraire, leur méconnaissance expose le médecin au prononcé d'une sanction disciplinaire. À l'opposé, dans un système d'incitations financières, à l'image de celui conçu par la convention nationale des médecins, le médecin est récompensé par la caisse primaire d'assurance maladie à la mesure de la conformation de ses actes aux «bonnes pratiques ».

On pourrait être tenté d'adhérer à cette promotion de la sanction positive. Mais ce serait oublier qu'elle repose sur une théorie différente des ressorts de l'action humaine. L'homme du code de déontologie est un être doué de raison, à ce titre supposé en capacité d'agir dans l'intérêt du patient ${ }^{51}$. Aussi est-ce cette capacité d'action qui appelle protection. Tel est pour la Cour de Justice de l'Union européenne le sens des dispositions qui visent à empêcher toute promotion commerciale des médicaments auprès des médecins prescripteurs. La figure de l'homme mû par son propre intérêt, maximisateur de ses utilités, sert au contraire de point d'appui aux développements des dispositifs incitatifs ${ }^{52}$. « L'incitation fait ainsi surgir dans la relation de soin un facteur jusque-là absent, ou tout au moins tenu à distance : l'intérêt individuel du médecin $»^{53}$.

La rémunération à la performance répond à une volonté de faire évoluer le mode de rémunération des professions de santé. Sans remettre en cause la pertinence de ce projet, l'irruption de l'intérêt individuel du médecin dans la prise de décisions relatives aux patients rend dubitatif quant à l'avenir de cette rétribution alors que d'autres formes sont concevables ${ }^{54}$.

Pour qui veut rendre compte de la nature et de l'ampleur des changements qui se sont produits, Robert Castel note dans l'un de ses derniers textes qu' « il ne faut pas craindre de réactualiser le sens des catégories que l'on emploie, de repérer les nouvelles $»^{55}$.

\footnotetext{
50. Ouvrage précité note (42).

51. Sur les fondements juridiques de la personne, A. SUPIOT, Homo juridicus. Essai sur la fonction anthropologique du droit, Paris, Éditions du Seuil, 2005, spécialement p. 45 et suivantes.

52. Sur la figure de l'homme intéressé, C. LAVAL, L'Homme économique. Essai sur les racines du néolibéralisme, Paris, Gallimard, «Nrf essais », 2007, 416 p.

53. A. Allouache, A.-S. Ginon et alii, article précité note (24).

54. Sur les limites de ce mode de rémunération, voir B. DORMONT, article précité note (24).

55. R. Castel, «Penser le changement : le parcours des années 1966-2010», in Changements et pensées du changement, sous la direction de R. CASTEL et C. MARTIN, Paris, La Découverte, 2011, p. 23.
} 
À son tour, Michel Miaille souligne comment «les juristes sont provoqués à renouveler plus que leurs mots, leur manière de penser $\$^{56}$. Cette invitation à reconsidérer les manières de penser est particulièrement bienvenue dans un domaine, tel que celui de la santé, où se déploie la raison statistique. Outre que seule cette attitude permet de saisir la dynamique de cette branche du droit, elle est aussi la meilleure façon de souligner quelles sont, en retour, les limites qu'un système juridique tel que le nôtre fixe aux innovations politiques.

56. M. MiAILLE, «La régulation dans les manuels de droit administratif », in Mélanges en l'honneur du professeur Jacques Chevallier, Paris, LGDJ, 2013, p. 467-480. 